\title{
Detecção de Abelhas Nativas em Colmeias em Campo Utilizando Visão Computacional
}

\author{
Rodolfo R. V. Leocádio ${ }^{1}$, Alan K. R. Segundo ${ }^{1}$, Jefferson R. Souza ${ }^{2}$, Juliana \\ Galaschi-Teixeira ${ }^{3}$, Paulo de Souza ${ }^{4}$, Gustavo Pessin ${ }^{1,5}$ \\ ${ }^{1}$ Programa de Pós-Graduação em Ciência da Computação \\ Universidade Federal de Ouro Preto (UFOP) - Ouro Preto, MG, Brasil \\ ${ }^{2}$ Faculdade de Computação \\ Universidade Federal de Uberlândia (UFU) - Uberlândia, MG, Brasil \\ ${ }^{3}$ Instituto Tecnológico Vale - Desenvolvimento Sustentável \\ Belém, PA, Brasil \\ ${ }^{4}$ School of Information and Communication Technology \\ Griffith University - Gold Coast, Queensland 4222, Australia \\ ${ }^{5}$ Instituto Tecnológico Vale - Mineração \\ Ouro Preto, MG, Brasil \\ \{rodolfo.leocadio, alankardek\}@ufop.edu.br, jrsouza@ufu.br, \\ \{juliana.teixeira, gustavo.pessin\}@itv.org, \\ paulo.desouza@griffith.edu.au
}

\begin{abstract}
Artificial intelligence approaches can, like computer vision, help improve the understanding behavior of bee species. Accurate detection of bees in the field is challenging. We investigate the use of the detector YOLO in the task of species recognition; with benefits from its speed and generalization. YOLO detected most bees present in the frames, with an effectiveness mAP of $99.5 \%$.

Resumo. Abordagens de inteligência artificial, como visão computacional, podem ajudar a entender melhor o comportamento de abelhas e ajudar no seu manejo. Detectar espécies de abelhas em campo é ainda um desafio para os métodos tradicionais. Neste trabalho, propõe-se o uso do detector YOLO justificado por sua capacidade, velocidade e generalização. Foi possível detectar a grande maioria das abelhas presentes nos frames, com mAP de 99,5\% de eficácia.
\end{abstract}

\section{Introdução}

Os insetos desempenham um papel fundamental na natureza incluindo polinização, herbivoria, detrivoria, ciclagem de nutrientes e fonte de alimento para outras espécies [Hallmann et al. 2017]. As abelhas nativas são polinizadores que se relacionam com variados alimentos vegetais consumidos pela humanidade [Arruda et al. 2018 (apud Klein et al. 2007)]. Polinizadores, particularmente insetos sociais como abelhas, desempenham um papel importante na agricultura mundial, pois melhoram a qualidade da colheita, e consequentemente, o rendimento financeiro das culturas [Kuan et al. 2018]. Além disso, elas contribuem ativamente na recuperação de ecossistemas degradados [Giannini et al. 
2020], podem ser utilizadas em biovetorização, tecnologia que envolve o uso de insetos como agentes de biocontrole [Macharia et al. 2020] e também podem desempenhar um papel crucial em projetos e programas de desenvolvimento sustentável [Giannini et al. 2020]. Perdas de biodiversidade entre abelhas nativas têm sido documentadas para as regiões tropicais [Sánchez-bayo e WyckhuysBorges 2019] e [Borges et al. 2020]. Os principais fatores responsáveis por esse declínio incluem: infecções disseminadas por parasitas e patógenos; falta de variabilidade genética; estresse devido a movimentação sazonal de colmeias para polinização de frutas e hortaliças; resíduos de pesticidas tóxicos encontrados no pólen e néctar ou aplicado a colmeias para controlar ácaros; baixo valor nutricional de agropaisagens dominadas por monoculturas (como milho, soja e algodão); e condições climáticas mais adversas das últimas décadas [Hallmann et al. 2017], [Sánchez-bayo e WyckhuysBorges 2019], [Gomes et al. 2020], [Filipiak 2018] e [Marstaller et al. 2019].

O declínio dos insetos resulta em efeitos adversos em ecossistemas. Assim, preservar sua abundância e diversidade são de grande importância para ecologia [Hallmann et al. 2017]. Indivíduos com pouco conhecimento entomológico não são capazes de distinguir categorias de insetos, bem como seus estágios de maturidade e comportamento. Desta forma, é necessário desenvolver abordagens mais rápidas e eficazes para resolver esses problemas [Xia et al. 2018]. As questões que devem ser abordadas no processo de reconhecimento e classificação de insetos são: (i) detectar rapidamente o animal de interesse posicionado em um fundo complexo; (ii) distinguir com precisão adequada as espécies de insetos com alta similaridade entre as classes; e (iii) identificar efetivamente padrões de interesse do comportamento das classes. Ferramentas que fazem uso de inteligência artificial podem ter uma relação mutualista com os demais métodos aplicados em ecologia. O monitoramento de colmeias pode ajudar no combate ao declínio das abelhas, relacionadas ao forrageamento, características do pólen, comportamento da colmeia e espécies invasoras.

Para ajudar no monitoramento das colmeias, este trabalho faz uso de um detector de objetos, um modelo de machine learning, baseado em visão computacional para detectar abelhas da espécie Melipona seminigra (Figura 1). Estudos atuais sobre recursos utilizados pelo gênero Melipona se relacionam com a ecologia de plantas nativas da região Amazônica [Pimentel et al. 2020].

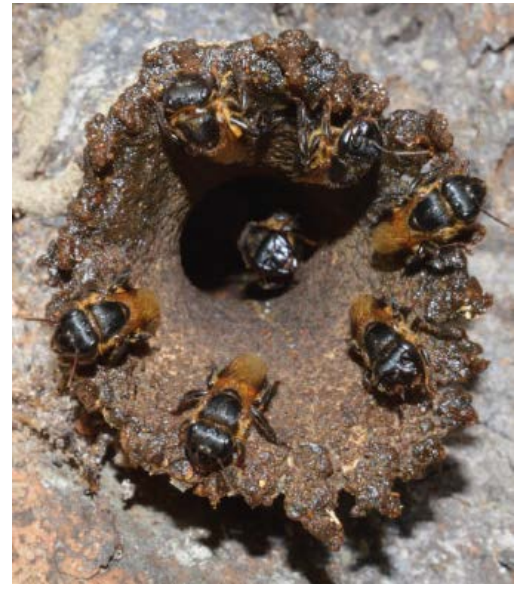

Figura 1. Abelha nativa da amazônia Melipona seminigra (Uruçu-boca-de-renda ou uruçu-preta) [Costa 2019]. 
Considerando a metodologia desenvolvida, neste trabalho de pesquisa admite-se a hipótese de que detectar e classificar essa espécie de abelha no campo é a tarefa inicial para implementação de um monitoramento de colmeias. A partir disso será possível implementar as demais fases (detecção de espécies invasoras, identificação do sentido dos movimentos das abelhas, contagem de insetos e identificação de pólen) que podem constituir um monitoramento inteligente das colmeias dessas espécies. A seguir são apresentados os trabalhos mais recentes relacionados a inteligência artificial auxiliando no manejo e conservação de insetos, bem como o método utilizado na detecção e classificação da Melipona seminigra. Os resultados obtidos são apresentados e discutidos, bem como trabalhos e perspectivas futuras.

\section{Trabalhos relacionados}

Alguns trabalhos desenvolvidos nos últimos três anos se destacam por tentar automatizar processos aplicados a Ciências Biológicas envolvendo a Classe Insecta do Filo Arthropoda (Reino Animalia). Técnicas de Inteligência Artificial (IA) utilizadas para identificar e contar insetos a partir de imagens podem resultar em uma alternativa para reduzir atividades manuais, e erros de origem humana [Júnior e Rieder 2020]. Nesse sentido, há uma tendência em utilizar abordagens de deep learning para o reconhecimento de insetos. Dentre elas, a técnica de detecção de objetos provou ser mais adequada e a abordagem Faster R-CNN tem sido mais utilizada para essa tarefa. Desta forma, tem sido possível identificar e contar insetos em plantas, folhas, fotografias, armadilhas e grãos [Júnior e Rieder 2020].

Outras abordagens como a combinação dos modelos VGG19 e Region Proposal Network (RPN) para reconhecimento e classificação de insetos no campo [Xia et al. 2018] também têm sido utilizadas. Estudos anteriores ainda incluem algumas outras espécies de insetos, como vespas e mariposas, classificadas com técnicas de machine learning.

Liu et al. (2019) desenvolveram um sistema chamado PestNet para detecção de pragas em campo. O monitoramento do número de espécies de pragas evita o uso indiscriminado de pesticidas que resultam em safras prejudiciais à saúde. A abordagem é satisfatória para a tarefa, porém seu melhor desempenho atingiu precisão de somente 75,46\% mean Average Precision (mAP). O equipamento de aquisição é baseado em armadilha de luz multiespectral e uma câmera HD, projetado de forma que espécimes de pragas capturadas pela armadilha em uma bandeja sejam fotografadas periodicamente com posterior limpeza da bandeja. O PestNet consiste de três etapas: extração de características, pesquisa de regiões de interesse (RoI) e previsão das pragas. Para isso, é utilizado um backbone com Convolutional Neural Network (CNN) para extrair mapas de recursos e Channel-Spatial Attention (CSA) para aprimoramento dos recursos. Em seguida, RPN e Position-Sensitive Score Map (PSSM) para fornecer regiões de pragas e previsão de pragas, respectivamente. Durante a fase de predição, os RoIs contextuais são apresentados como informações contextuais para melhorar a precisão da detecção. NonMaxima Supression (NMS) é adotado como técnica para eliminar sobreposição de caixas delimitadoras.

Algoritmos para detecção de objetos baseado no Faster R-CNN foram aplicados para identificar insetos em grãos armazenados misturados com finos (fines), materiais estranhos (foreign materials), impurezas (dockages) e grãos quebrados (broken grains); sob diferentes condições de iluminação [Shen et al. 2018]. Os procedimentos foram 
realizados em laboratório. As etapas de detecção do objeto alvo foram: adquirir as regiões propostas na imagem pela RPN, obter caixas candidatas por NMS, mapear as caixas candidatas como mapas de características, classificar essas regiões e usar o NMS para evitar caixas candidatas sobrepostas.

Outra abordagem com grãos propõe monitorar pragas de arroz em tempo real com objetivo de realizar um manejo dessas pragas [Qing et al. 2020]. Cinco espécies no campo são identificadas e contadas, três grandes ( 8 a $15 \mathrm{~mm}$ ) com 88,9\% mAP e duas pequenas (3 a $5 \mathrm{~mm}$ ) com 90,4\% mAP. A metodologia de captura das pragas é similar a proposta por Liu et al. (2019), mas as imagens dos insetos são enviadas para um servidor na nuvem que intermedia a comunicação entre a armadilha e os dispositivos de controle. As imagens são pré-processadas: remoção de fundo, remoção de ruído e segmentação de insetos que se tocam. As regiões contendo uma praga alvo passam por um classificador CNN de insetos grandes (ResNet-152) ou por um classificador CNN de insetos pequenos (ResNet18).

Concomitantemente, trabalhos envolvendo a Família Apidae da Ordem Hymenoptera também têm sido desenvolvidos. Para identificar espécies de abelhas com machine learning interpretando dados de forrageamento, Arruda et al. (2018) monitoraram abelhas com etiquetas RFID coladas em seu tórax. Os dados gerados pelos RFID são pré-processados em vetores de recursos. Três métodos de machine learning são empregados, mas somente o modelo Random Forest consegue atender ao objetivo, para diferentes configurações de vetores de recursos. Desta forma foi possível classificar duas espécies de abelhas amazônicas por meio de seu padrão de forrageamento. Nessa mesma linha de pesquisa, ocorreram investigações como uma forma de melhorar previsões da atividade das abelhas por meio de Recurrent Neural Networks (RNN), com abordagem relacionada ao comportamento das abelhas [Gomes et al. 2020]. Também utiliza etiquetas RFID coladas em abelhas em conjunto com dados de temperatura, pressão barométrica e irradiância solar. Para isso, definem uma arquitetura RNN que tem o melhor desempenho na previsão do comportamento das abelhas, mostram que diferentes tamanhos de entrada impactam na precisão da previsão e mostram como algoritmos para seleção de atributos e análise de correlação podem ajudar a melhorar a precisão da previsão.

Existem ainda sistemas com deep learning para monitoramento automatizado de colmeias de abelhas [Marstaller et al. 2019]. Neste caso, as abelhas são filmadas na entrada da colmeia. O processamento responsável por localizar e rastrear as abelhas são executados na borda. Amostras de abelhas individuais são transferidos para a nuvem, onde o processo de inferência sobre a saúde das abelhas é executado (DeepBees). Um módulo chamado de Genus distingue entre abelhas e vespas. Dessa forma, informações sobre ataques e invasões são coletados. O módulo Pollen detecta pólen nas abelhas, gerando e agregando medidas da nutrição na colmeia e identificando carências de comida. O módulo Pose prevê a localização de 32 pontos-chave no inseto, benéfico para identificar anomalias de comportamento e no monitoramento da saúde e higiene da colmeia. O módulo Classification pontua probabilidades para um total de quatro classes mutuamente exclusivas: abelhas operárias com pólen, sem pólen, zangões e abelhas mortas. Também é incluído um módulo Decoder para aprendizado estrutural. Contudo, ainda existem desafios na precisão da detecção de pólen, no mapeamento direto de atividades, doenças ou infestação de ácaros devido à falta de dados. 


\section{Metodologia}

Detectar objetos é uma tarefa de visão computacional que lida com a detecção de instâncias de objetos semânticos de uma determinada classe em imagens e vídeos digitais. Alguns métodos podem ser utilizados para esta tarefa como: (i) Deformable parts models usam uma abordagem com janelas deslizantes para detecção de objetos; (ii) R-CNN e suas variantes usam propostas de região, como a Busca Seletiva, para encontrar objetos nas imagens; (iii) Deep MultiBox treina uma CNN para prever RoI; (iv) OverFeat requer pós-processamento significativo para produzir detecções coerentes; (v) MultiGrasp prevê uma única região na imagem contendo um objeto; e (vi) YOLO (You Only Look Once) unifica os componentes de detecção de objetos em uma única rede neural [Redmon et al. 2015].

Este trabalho utiliza o detector de código aberto YOLO devido a sua velocidade de detecção ser consideravelmente rápida, por analisar globalmente a imagem ao fazer previsões e por aprender representações generalizáveis dos objetos. Isso somente é possível devido a detecção de quadros ser tratado como um problema de regressão, não precisando de um pipeline complexo [Redmon et al. 2015].

A versão 4 do YOLO [Bochkovskiy et al. 2020] recebe as imagens com formato de $(320,320,3)$ a $(608,608,3)$ [Redmon e Farhadi 2016] em sua entrada. O Backbone utiliza a CNN CSPDarknet53 como extrator de características. Spatial Pyramid Pooling (SPP) e Path Aggregation Network (PAN) compõem o Neck. O bloco SPP sobre o CSPDarknet53 aumenta significativamente o campo receptivo e separa as características de contexto mais significativas, sem causar redução significativa da velocidade. Já o PAN é o método de segmentação e agregação de parâmetros do backbone para diferentes níveis do detector. A Head é composta pela versão 3 do YOLO [Redmon e Farhadi 2018], baseado em âncoras [Redmon e Farhadi 2016], que utiliza classificadores logísticos independentes ao invés do softmax. Este conjunto compõem a arquitetura YOLO.

Uma imagem pré-processada contendo o objeto, como o exemplo da Figura 2a, entra na rede neural convolucional representada na Figura $2 b$, que por sua vez divide a imagem em um grid de 19x19 células (Figura 2c). Cada uma dessas células é responsável por fazer a predição de 5 caixas delimitadoras, detalhadas na Figura 2d, caso haja mais de um objeto naquela célula.

Cada caixa delimitadora (Figura 2d) pode ser descrita com uma pontuação de confiança (pc), que indica a probabilidade de existir um objeto naquela caixa delimitadora; centro (bx, by), largura (bw) e altura (bh) da caixa delimitadora e; classe (c), um valor de probabilidade para cada uma das classes possíveis do objeto. pc e c são combinados em uma pontuação final para gerar as caixas delimitadoras que contém 0 objeto detectado, como exemplificado na Figura 2e. NMS é aplicada para remover caixas com baixa probabilidade de conter um objeto e também caixas que possuem uma área compartilhada, restando somente uma caixa delimitadora contendo o objeto identificado (Figura 2f).

O pré-processamento das imagens é feito utilizando a biblioteca OpenCV ${ }^{1}$ no Python $^{2}$. As imagens são normalizadas com padrão de dados float 32, redimensionadas para o tamanho (416x416) preservando sua proporção, convertidas para o padrão RGB e

\footnotetext{
${ }^{1}$ https://opencv.org/

2 https://www.python.org/
} 
transformadas em um vetor com 4 dimensões com a forma (Lotes, Canais, Largura, Altura), compatível com a entrada do YOLO.

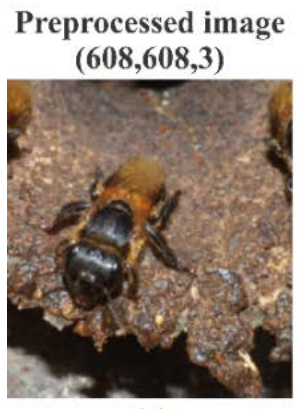

(a)

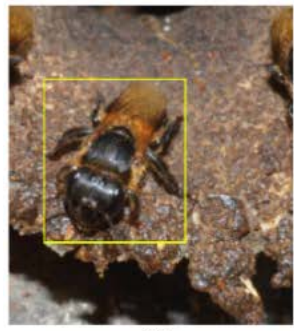

(f)
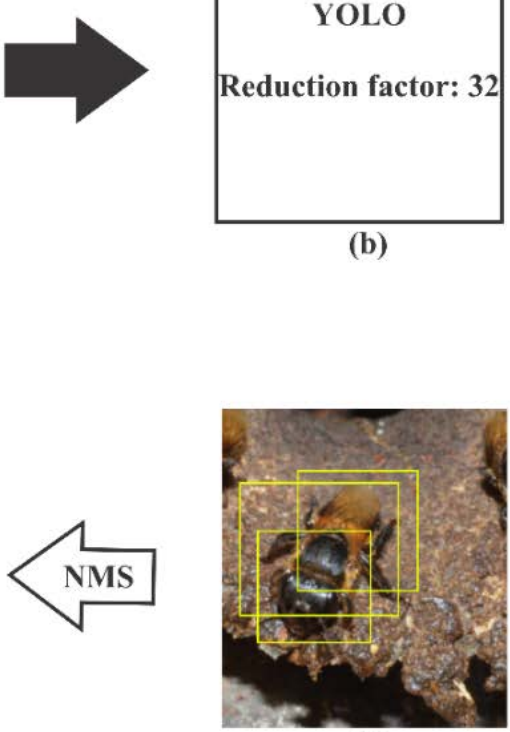

(e)

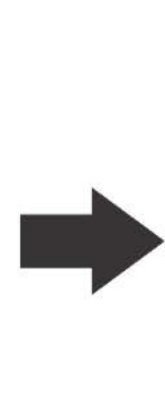

Encoding $(19,19,5,85)$

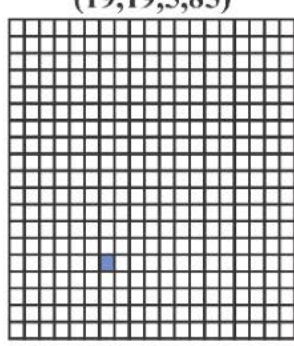

(c)
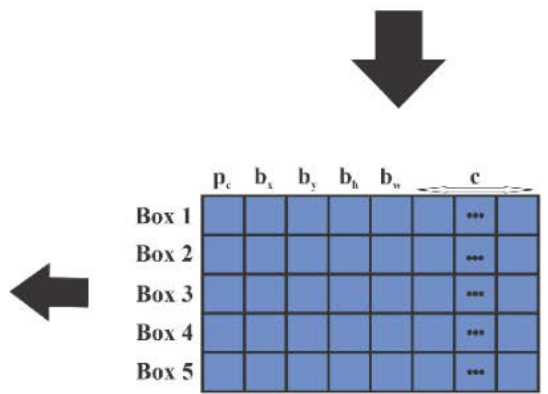

(d

Figura 2. Método de previsão de abelhas em imagens.

Vídeos de colmeias da espécie em questão foram gravados no campo com dispositivos móveis, com tamanho de 1280x720 em condições adequadas de iluminação. Os vídeos apresentam a movimentação na entrada da colmeia, ocasionando em diferentes poses e inúmeras situações em que desejam-se identificar as abelhas. Para cada frame contendo uma pose ainda não marcada, utilizou-se o LabelImg $^{3}$ (software livre) para gerar anotações desses frames. As anotações contêm um número correspondente à classe do objeto seguido das coordenadas das caixas delimitadoras contendo abelhas. O Dataset para o treinamento foi composto com 459 imagens, sendo 368 imagens para treinamento e 91 imagens para validação.

O treinamento pode ser realizado com o YOLO, caso deseje uma melhor precisão na detecção ou com o YOLO-Tiny (utilizado neste trabalho), caso deseje maior velocidade de detecção. Lotes de 64 imagens são gerados para cada época de treinamento. Foram utilizadas 2000 épocas para treinamento do modelo que identifica somente uma classe “Abelhas”. O treinamento é realizado no Google Colaboratory ${ }^{4}$, um serviço gratuito na nuvem voltado a pesquisas de machine learning e Inteligência Artificial. A disponibilização de GPU foi a principal justificativa na utilização do Colaboratory. Pesos pré-treinados no conjunto de dados COCO [Redmon e Farhadi 2016] foram utilizados para inicializar a rede.

As métricas utilizadas para avaliar o modelo são: Loss - Quanto menor, melhor a acurácia do modelo; Average Precision (AP) - Acurácia do detector para cada classe; mean Average Precision (mAP) - Média do AP; Precision - Informa o quanto o modelo

\footnotetext{
${ }^{3}$ https://github.com/tzutalin/labelImg

${ }^{4}$ https://colab.research.google.com
} 
está certo quando realizou previsões; Recall - Informa o quanto o modelo classificou corretamente; e Intersecion over Union (IoU) - Avalia o desempenho do detector, ou seja, se ele encontrou a classe e a localização do objeto (caixas delimitadoras).

O IoU foi predefinido para um limite de 0,5 ao classificar a previsão. Se IoU $\geq$ 0,5, classifica-se a detecção do objeto como True Positive (TP). Um TP ocorre quando o detector prevê as classes corretamente. Já IoU < 0,5 indica uma detecção errada, logo, classifica-se como False Positive (FP). Um FP é classificado como erro do detector e ocorre quando o classificador erra a previsão. Um True Negative (TN) ocorre quando o detector prevê que não há objeto de interesse na região em questão e realmente não há. E False Negative (FN), também classificado como erro do detector, ocorre quando o classificador prevê que não há objeto de interesse na região em questão, porém, há.

\section{Resultados e discussão}

O resumo do processo de treinamento do detector pode ser visualizado na Figura 3. Tratase de um gráfico de Loss em função das épocas do treinamento. A partir do gráfico, foi obtido um valor para Loss de 0,1966 e mAP de 99,5\%. Vale ressaltar que, neste caso, AP é igual a mAP, pois foi treinado o modelo com só uma classe. Para um dataset pequeno que contém poucas classes, o valor final de Loss pode cair até a segunda casa decimal após a virgula. As abelhas observadas no campo exibem inúmeras poses que devem ser consideradas durante o treinamento, portanto, a Loss obtida está entre os valores aceitáveis para a aplicação. A acurácia do detector para a classe Melipona seminigra (Figura 1) apresentou resultados que surpreenderam os autores, porém deve ser monitorado minunciosamente com a adição de novas classes.

O valor de IoU é $81,81 \%$, ou seja, o detector foi capaz de localizar as abelhas. As caixas delimitadoras traçadas enquadram as Melipona seminigra de forma que possam ser identificadas. O modelo está correto em 98\% (Precision) das vezes que realizou as detecções e em 99\% (Recall) das vezes que classificou. Isso indica que ocorrem alguns erros durante as detecções e que ocorrem erros mínimos na classificação das detecções. O detalhe das observações é apresentado na Tabela 1.

Tabela 1. Matriz de confusão do treinamento do modelo.

\begin{tabular}{ccc}
\hline YOLO & Com abelha & Sem abelha \\
\hline Com abelha & $431(\mathrm{TP})$ & $9(\mathrm{FP})$ \\
\hline Sem abelha & $4(\mathrm{FN})$ & $473(\mathrm{TN})$ \\
\hline
\end{tabular}

A matriz de confusão indica que o sistema acerta a grande maioria das abelhas presentes nos frames. Os resultados exibiram métricas relativamente altas, sugerindo que os erros ocorridos são pouco significativos. Também foi possível notar que a principal fonte de erro são as localizações incorretas detectadas, seguindo o mesmo padrão descrito em Redmon et al. (2015).

O maior desafio nesse tipo de implementação é conseguir condições e dados provenientes das espécies que se deseja detectar e classificar em campo para compor o dataset de treinamento. Intempéries comuns em ambientes naturais dificultam a utilização e manutenção do hardware. Para diversificar os dados para o treinamento são necessárias prolongadas horas de gravação na colmeia, e posterior mineração desses dados com o objetivo de reconhecer ataques e inúmeras poses diferentes com e sem pólen. 


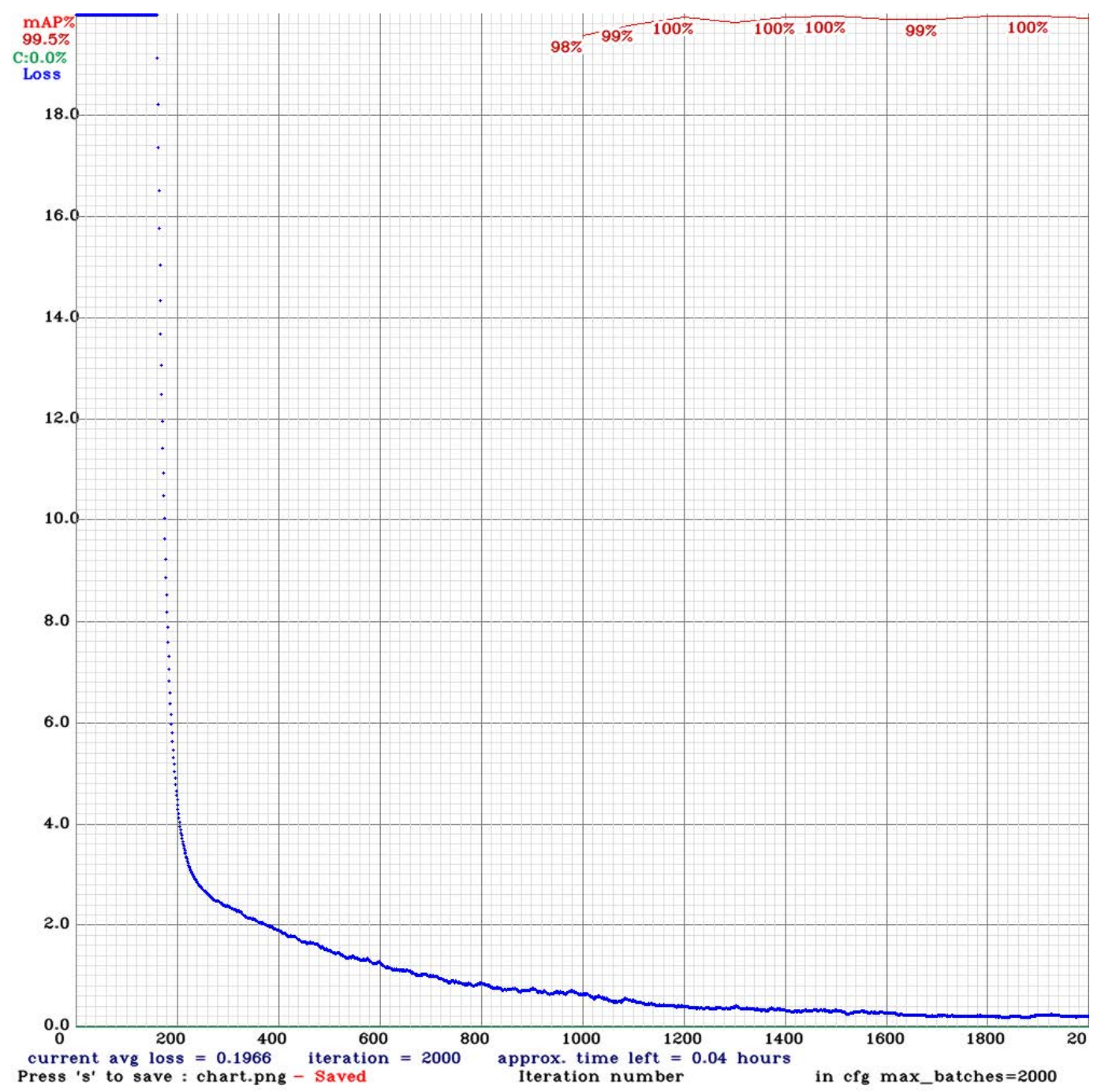

Figura 3. Loss e mAp do treinamento do detector de abelhas em campo.

O próximo passo é desenvolver um trabalho de monitoramento completo de colmeias de Melipona seminigra, semelhante ao que foi feito por Marstaller et al. (2019). O monitoramento deve englobar detecção de espécies invasoras nativas da Amazônia como outras espécies de abelhas, formigas, aranhas, vespas, dentre outros como forídeos. Deve-se identificar o sentido dos movimentos das abelhas, entrada e saída da colmeia, e realizar a contagem das mesmas, com o objetivo de facilitar trabalhos semelhantes a Arruda et al. (2018), de Souza et al. (2018) e Gomes et al. (2020). Por fim, pretende-se também identificar o pólen, para agregar monitoramento da nutrição e carências de alimentos na colmeia.

\section{Agradecimentos}

Os autores agradecem a Luciano Costa (ITV-DS), Helder Arruda (Unisinos) e Vera Lúcia Imperatriz-Fonseca (USP) por diversas e frutíferas discussões e também pelo apoio na obtenção dos dados ambientais. Os autores agradecem a Renato Hidaka (UFPA) pelo apoio com os métodos computacionais. Esta pesquisa recebeu apoio financeiro da Coordenação de Aperfeiçoamento de Pessoal de Nível Superior (CAPES) - Código de financiamento 001. 


\section{Referências}

Arruda, H., Imperatriz-fonseca, V., De Souza, P. e Pessin, G. (2018) “Identifying Bee Species by Means of the Foraging Pattern Using Machine Learning”, in 2018 International Joint Conference on Neural Networks (IJCNN), p. 1-6.

Bochkovskiy, A., Wang, C. e Liao, H. M. (2020) "YOLOv4: Optimal Speed and Accuracy of Object Detection”, Cornell Univ., no. April. arXiv:2004.10934v1

Borges, R. C., Padovani, K., Imperatriz-Fonseca, V. L. e Giannini, T. C. (2020) “A dataset of multi-functional ecological traits of Brazilian bees”, Sci. Data, vol. 7, p. 1-9. doi: https://doi.org/10.1038/s41597-020-0461-3

Costa, L. (2019) Guia Fotográfico de Identificação de Abelhas Sem Ferrão, para resgate em áreas de supressão florestal. Belém: Brasil.

de Souza, P., Marendy, P., Barbosa, K., Budi, S., Hirsch, P., Nikolic, N., Gunthorpe, T. Pessin, G., Davie, A. (2018) "Low-Cost Electronic Tagging System for Bee Monitoring”, Sensors vol.18 2124. doi: 10.3390/s18072124

Filipiak, M. (2018) “A Better Understanding of Bee Nutritional Ecology Is Needed to Optimize Conservation Strategies for Wild Bees - The Application of Ecological Stoichiometry”, Insects, vol. 9, no. July, p. 1-13. doi: 10.3390/insects9030085

Giannini, T. C., Costa, W. F., Borges, R. C., Miranda L., Costa, C. P. W., Saraiva, A. M. e Fonseca, V. L. I. (2020) "Climate change in the Eastern Amazon: crop-pollinator and occurrence-restricted bees are potentially more affected”, Reg. Environ. Chang., vol. 20, p. 1-12. doi: https://doi.org/10.1007/s10113-020-01611-y

Gomes, P. A. B., Suhara, Y., Nunes-Silva, P., Costa, L., Arruda, H., Venturieri, G., Imperatriz-Fonseca, V. L., Pentland, A., Souza, P. e Pessin, G. (2020) “An Amazon stingless bee foraging activity predicted using recurrent artificial neural networks and attribute selection", Nature research, vol. 10, pp. 1-12. doi: https://doi.org/10.1038/s41598-019-56352-8

Hallmann, C. A., Sorg, M., Jongejans, E., Siepel, H., Hofland, N., Schwan, H., Stenmans, W., Müller, A., Sumser, H., Hörren, T., Goulson, D. e de Kroon, H. (2017) "More than 75 percent decline over 27 years in total flying insect biomass in protected areas”, PLoS One, vol. 12, no. October, p. 18-22. doi: https://doi.org/10.1371/journal.pone.0185809

Júnior, T. D. C. and Rieder, R. (2020) “Automatic identification of insects from digital images: A survey”, Comput. Electron. Agric., vol. 178, no. April, p. 105784. doi: https://doi.org/10.1016/j.compag.2020.105784

Kuan, A. C., Grandi-Hoffman, G., Curry, R. J., Garber, K. V., Kanarek, A. R., Snyder, M. N., Wolfe, K. L. e Purucker, S. T. (2018) "Sensitivity analyses for simulating pesticide impacts on honey bee colonies”, Ecol. Modell., vol. 376, no. February, p. 1527. doi: https://doi.org/10.1016/j.ecolmodel.2018.02.010

Liu, L., Wang, R., Xie, C., Yang, P., Wang, F., Surdiman, S. e Liu, W. (2019) "PestNet: An End-to-End Deep Learning Approach for Large-Scale Multi-Class Pest Detection and Classification”, IEEE Access, vol. 7, p. 45301-45312. doi: 10.1109/ACCESS.2019.2909522 
Macharia, J. M., Gikungu, M. W., Karanja, R. e Okoth, S. (2020) "Managed bees as pollinators and vectors of bio control agent against grey mold disease in strawberry plantations”, African J. Agric. Res., vol. 16, no. 12, p. 1674-1680. doi: 10.5897/AJAR2020.15203

Marstaller, J., Tausch, F. e Stock, S. C. (2019) “DeepBees - Building and Scaling Convolutional Neuronal Nets For Fast and Large-Scale Visual Monitoring of Bee Hives”, in ICCV Workshop, October. doi: 10.1109/ICCVW.2019.00036

Pimentel, A. D. A., Absy, M. L., Rech, A. R. e de Abreu, V. H. R. (2020) "Pollen sources used by Frieseomelitta Ihering 1912 (Hymenoptera: Apidae: Meliponini) bees along the course of the Rio Negro, Amazonas, Brazil”, Acta Bot. Brasilica, vol. 34, no. June, p. 371-383. doi: 10.1590/0102-33062019abb0391

Qing, Y., Jin, F., Jian, T., Wei-gen, X., Xu-hua, Z., Bao-jun, Y., Jun, L., Yi-ze, X., Bo1, Y., Shu-zhen, W., Nai-yang, K. e Li-jun, W. (2020) "Development of an automatic monitoring system for rice light-trap pests based on machine vision”, J. Integr. Agric., vol. 19, no. February, p. 2500-2513. doi: 10.1016/S2095-3119(20)63168-9

Redmon, J. e Farhadi, A. (2016) "YOLO9000: Better, Faster, Stronger”, Cornell Univ., no. December. arXiv:1612.08242v1

Redmon, J. e Farhadi, A. (2018) “YOLOv3: An Incremental Improvement”, Cornell Univ., no. April. arXiv:1804.02767v1

Redmon, J., Divvala, S., Girshick, R. and Farhadi, A. (2015) "You Only Look Once: Unified, Real-Time Object Detection”, Cornell Univ., no. May. arXiv:1506.02640v5

Sánchez-bayo, F. e Wyckhuys, K. A. G. (2019) "Worldwide decline of the entomofauna: A review of its drivers”, Biol. Conserv., vol. 232, no. September, p. 8-27. doi: https://doi.org/10.1016/j.biocon.2019.01.020

Shen, Y., Zhou, H., Li, J., Jian, F. e Jayas, D. S. (2018) “Detection of stored-grain insects using deep learning”, Comput. Electron. Agric., vol. 145, no. October, p. 319-325. doi: https://doi.org/10.1016/j.compag.2017.11.039

Xia, D., Chen, P., Wang, B., Zhang, J. e Xie, C. (2018) "Insect Detection and Classification Based on an Improved Convolutional Neural Network", Sensors, vol. 18, no. November, p. 1-12. doi: 10.3390/s18124169 